\title{
General relationship between contact stiffness, contact depth, and mechanical properties for indentation in linear viscoelastic solids using axisymmetric indenters of arbitrary profiles
}

\author{
Yang-Tse Cheng ${ }^{\text {a) }}$ \\ Materials and Processes Laboratory, General Motors Research and Development Center, Warren, Michigan \\ 48090 \\ Che-Min Cheng \\ Institute of Mechanics, Chinese Academy of Sciences, Beijing 100080, People's Republic of China
}

(Received 22 February 2005; accepted 22 July 2005; published online 9 September 2005)

\begin{abstract}
We derive a relationship between the initial unloading slope, contact depth, and the instantaneous relaxation modulus for indentation in linear viscoelastic solids by a rigid indenter with an arbitrary axisymmetric smooth profile. Although the same expression is well known for indentation in elastic and in elastic-plastic solids, we show that it is also true for indentation in linear viscoelastic solids, provided that the unloading rate is sufficiently fast. Furthermore, the same expression holds true for both fast loading and unloading. These results should provide a sound basis for using the relationship for determining properties of viscoelastic solids using indentation techniques. (C) 2005 American Institute of Physics. [DOI: 10.1063/1.2048820]
\end{abstract}

Instrumented indentation is playing an increasing role in the study of small-scale mechanical behavior of "soft" matters, such as polymers, composites, biomaterials, and food products. Many of these materials exhibit viscoelastic behavior, especially at elevated temperatures. Modeling of indentation into viscoelastic solids thus forms the basis for analyzing indentation experiments in these materials. Theoretical studies of contacting linear viscoelastic bodies became active since the mid 1950 s by the work of Lee, ${ }^{1}$ Radok, ${ }^{2}$ Lee and Radok, ${ }^{3}$ Hunter, Graham, ${ }^{4,6}$ Yang, and Ting. ${ }^{8,9}$ In recent years, a number of authors have extended the early work to the analysis of indentation measurements in viscoelastic solids. ${ }^{10-16}$

One of the widely used approaches is to obtain the elastic modulus from the initial unloading slope (Fig. 1), $d F / d h$, using the well-known relationship, ${ }^{17-21}$

$$
\frac{d F}{d h}=\frac{4 G}{1-\nu} a=\frac{2 E}{\sqrt{\pi}\left(1-\nu^{2}\right)} \sqrt{A},
$$

where $G$ is the shear modulus, $E=2 G /(1+\nu)$ is Young's modulus, $\nu$ is Poisson's ratio, $a$ is the contact radius, and $A=\pi a^{2}$ is the contact area. Equation (1) can be derived from the theory for elastic contacts between flat surfaces and spheres, ${ }^{22}$ flat punches, ${ }^{22}$ and conical punches. ${ }^{23}$ More generally, Sneddon has derived expressions for load, displacement, and contact depth for elastic contacts between a rigid, axisymmetric punch with an arbitrary smooth profile and an elastic half space. ${ }^{24}$ Using Sneddon's results, Pharr, Oliver, and Brotzen ${ }^{18}$ showed that Eq. (1) holds true for rigid indenters of arbitrary smooth profiles indenting elastic solids. Equation (1) has also been applied to indentation experiments where plastic deformation occurs. Doerner and Nix ${ }^{17}$ suggested that if the area in contact remains constant during initial unloading, the elastic behavior might be modeled as that of a blunt punch indenting an elastic solid. Oliver and

a) Author to whom correspondence should be addressed; electronic mail: yang.t.cheng@gm.com
Pharr ${ }^{19}$ pointed out that Eq. (1) can be used even when the contact area between the indenter and the solid changes continuously as the indenter is withdrawn and the indenter does not behave like a flat punch. We have recently shown that Eq. (1) is true for indentation in elastic-plastic solids with or without work hardening and residual stress. ${ }^{25}$ On the other hand, a "nose" (see Fig. 1) is often observed in the unloading curve when the unloading rate is slow for load-controlled indentation in viscoelastic solids. ${ }^{20,21}$ As a result, a nonphysical, negative Young's modulus is predicted according to Eq. (1). Recently, Lu et al., ${ }^{26}$ and Kumar and Narasimhan ${ }^{27}$ have suggested that Eq. (1) may not be applicable to indentation in viscoelastic solids even in the absence of a nose on the unloading curves. In this letter, we examine the validity of Eq. (1) for indentation in viscoelastic solids.

We consider a rigid, smooth, frictionless, axisymmetric indenter of arbitrary shape, $f(r)$, (Fig. 2) indenting a viscoelastic solid that can be described by the following constitutive relationships ${ }^{28,29}$ between deviatoric stress and strain, $s_{i j}$ and $d_{i j}$, and between dilatational stress and strain, $\sigma_{i i}$ and $\varepsilon_{i i}$ :

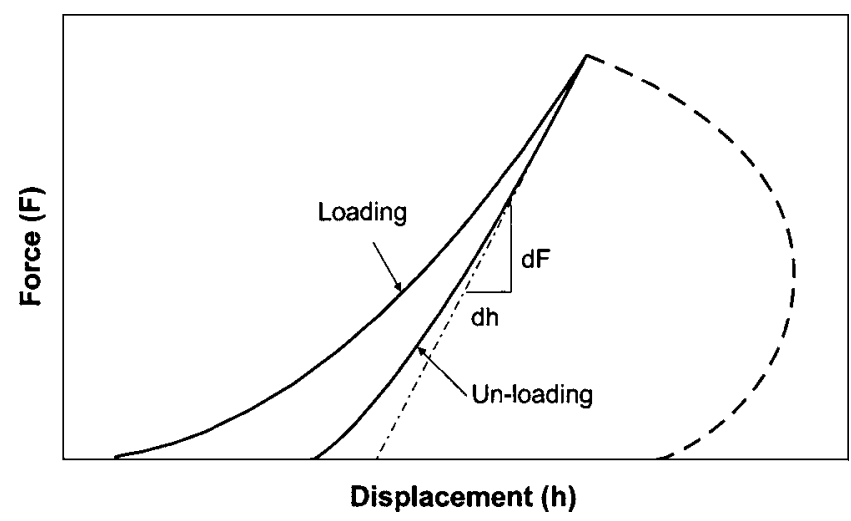

FIG. 1. Illustration of load-controlled indentation curves in viscoelastic solids. When the unloading rate is low, a nose is often observed in the unloading curve, resulting in a negative initial unloading slope. When the unloading rate is high, the initial unloading slope is positive. 


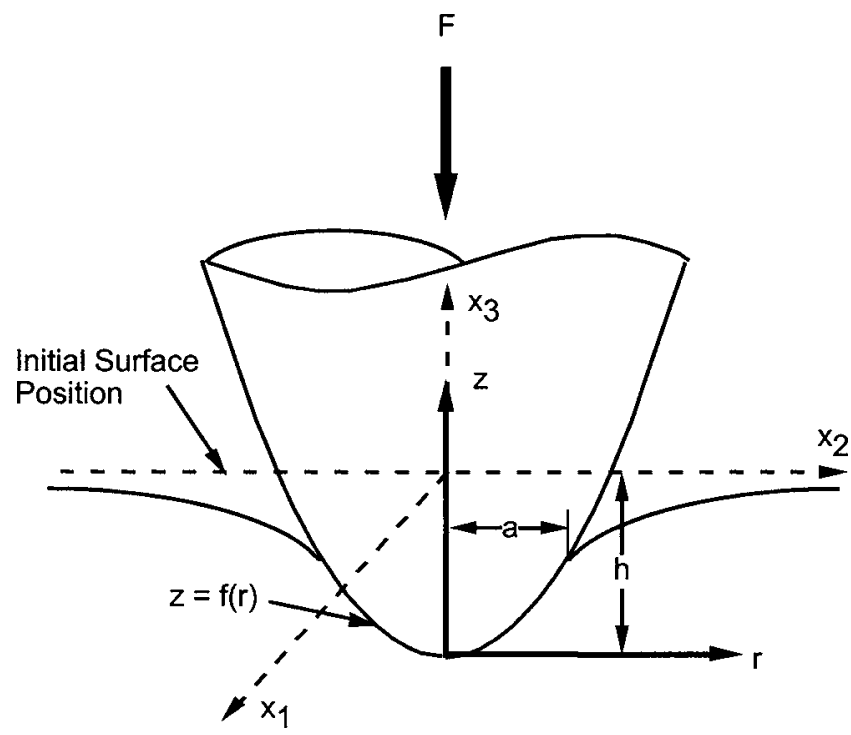

FIG. 2. Illustration of surface deformation by an axisymmetric indenter.

$$
\begin{aligned}
& s_{i j}(t)=2 \int_{0}^{t} G(t-\tau) \frac{\partial d_{i j}(\tau)}{\partial \tau} d \tau \\
& \sigma_{i i}(t)=3 \int_{0}^{t} K(t-\tau) \frac{\partial \varepsilon_{i i}(\tau)}{\partial \tau} d \tau,
\end{aligned}
$$

where $G(t)$ is the relaxation modulus in shear and $K(t)$ is the relaxation modulus in dilatation. The time dependent Young's modulus and Poisson's ratio are then given by $E(t)=[9 K(t) G(t)] /[3 K(t)+G(t)]$ and $\nu(t)=[E(t) / 2 G(t)]-1$, respectively.

When $G(t), K(t)$, and $\nu(t)$ are time independent, Eq. (2) reduces to the ones for elastic solids. The corresponding indentation problem has been solved previously, for example, by Sneddon, ${ }^{24}$ for the contact depth and indenter displacement relationship

$$
h=\int_{0}^{1} \frac{f^{\prime}(x)}{\sqrt{1-x^{2}}} d x
$$

and for the load and displacement relationship

$$
F=\frac{4 G a}{1-\nu} \int_{0}^{1} \frac{x^{2} f^{\prime}(x)}{\sqrt{1-x^{2}}} d x,
$$

where $x=r / a$. Using these relationships, Pharr, Oliver, and Brotzen ${ }^{18}$ derived Eq. (1) for rigid indenters of arbitrary smooth profiles indenting purely elastic solids.

Applying the theories developed by Lee and Radok, ${ }^{3}$ Graham, ${ }^{5}$ and Ting $^{8}$ to the problem of indentation in viscoelastic solids and assuming time independent Poisson's ratio, we can write

$$
\begin{aligned}
& h(t)=\int_{0}^{1} \frac{f^{\prime}(x)}{\sqrt{1-x^{2}}} d x \\
& F(t)=\frac{4}{1-\nu} \int_{0}^{t} G(t-\tau) \frac{d}{d \tau}\left[a(\tau) \int_{0}^{1} \frac{x^{2} f^{\prime}(x)}{\sqrt{1-x^{2}}} d x\right] d \tau,
\end{aligned}
$$

where $x=r / a(t)$.

Equations (5) and (6) become the familiar equations for conical indentation in linear viscoelastic solids, where $z$ $=f(x)=(a \tan \alpha) x$ and $\alpha$ is the indenter half angle. Specifically, the relationship between contact depth, $h_{c}(t)$, and the indenter displacement is given by, using Eq. (5)

$$
h(t)=\frac{\pi}{2} a(t) \tan \alpha=\frac{\pi}{2} h_{c}(t)
$$

and that between force and displacement is given by, using Eq. (6)

$$
F(t)=\frac{4}{\pi} \frac{\cot \alpha}{(1-\nu)} \int_{0}^{t} G(t-\tau) \frac{d h^{2}(\tau)}{d \tau} d \tau
$$

Likewise, the equations for spherical indentation in linear viscoelastic solids, where $f(x)=1 / 2(a x)^{2} / R$ and $R$ is the indenter radius, are given by

$$
\begin{aligned}
h(t) & =\frac{a^{2}(t)}{R}=2 h_{c}(t), \\
F(t) & =\frac{8}{3(1-\nu) R} \int_{0}^{t} G(t-\tau) \frac{d a^{3}(\tau)}{d \tau} \\
& =\frac{8 \sqrt{R}}{3(1-\nu)} \int_{0}^{t} G(t-\tau) \frac{d h^{3 / 2}(\tau)}{d \tau} d \tau .
\end{aligned}
$$

Equations (7)-(10) are special cases of more general expressions derived by Graham ${ }^{5}$ and Ting. ${ }^{8}$ They showed that Eqs. (7)-(10) are valid when the contact area is a monotonically increasing function of time. The equations for unloading where the contact area decreases monotonically have also been derived, ${ }^{5,8}$ though they are considerably more complicated. However, we have recently demonstrated that Eqs. (7) and (8) and Eqs. (9) and (10) can be used to evaluate the initial unloading slope for conical ${ }^{16,30}$ and spherical indentation $^{31}$ in viscoelastic solids, respectively. In the following, we use Eqs. (5) and (6) to derive the equation for initial unloading slopes for arbitrary indenter profiles.

Suppose unloading takes place at $t=t_{m}$ with a constant unloading rate of $d h(t) /\left.d t\right|_{t_{m}^{+}}=-v_{h}$, we have, using Eq. (6) for $0 \leqslant t \leqslant t_{m}+\Delta t$ and $\Delta t \rightarrow 0$

$$
\frac{F\left(t_{m}+\Delta t\right)-F\left(t_{m}\right)}{\Delta t}=\frac{4}{1-\nu}\left\{\left.\int_{0}^{t_{m}} \frac{d G}{d \eta}\right|_{\eta=t_{m}-\tau} \frac{d}{d \tau}\left[a(\tau) \int_{0}^{1} \frac{x^{2} f^{\prime}(x)}{\sqrt{1-x^{2}}} d x\right] d \tau+G(0) \frac{d}{d \tau}\left[a(\tau) \int_{0}^{1} \frac{x^{2} f^{\prime}(x)}{\sqrt{1-x^{2}}} d x\right]\right\} .
$$


Using Eq. (5), the derivative in the second term on the righthand side becomes

$$
\begin{aligned}
\frac{d}{d \tau} a(\tau) \int_{0}^{1} \frac{x^{2} f^{\prime}(x)}{\sqrt{1-x^{2}}} d x= & a(\tau) \frac{d h(\tau)}{d \tau}+\frac{d a(\tau)}{d \tau} h(\tau) \\
& -\frac{d a(\tau)}{d \tau} \int_{0}^{1} \sqrt{1-x^{2}} f^{\prime}(x) d x \\
& -a(\tau) \frac{d}{d \tau} \int_{0}^{1} \sqrt{1-x^{2}} f^{\prime}(x) d x .
\end{aligned}
$$

We now show that the last three terms on the right-hand side of Eq. (12) cancel each other. Using $x=r / a$ and $d x=d r / a$, and the fundamental theorem of calculus, we obtain

$$
\begin{aligned}
& \frac{d}{d \tau} \int_{0}^{1} \sqrt{1-x^{2}} \frac{d f(x)}{d x} d x \\
& \quad=\int_{r=0}^{r=a(\tau)} \frac{\partial}{\partial \tau}\left(\sqrt{1-\left(\frac{r}{a(\tau)}\right)^{2}}\right) \frac{d f(r)}{d r} d r .
\end{aligned}
$$

After evaluating the partial derivative in the integrand and using $x=r / a$, we have

$$
\begin{aligned}
\frac{d}{d \tau} \int_{0}^{1} \sqrt{1-x^{2}} \frac{d f(x)}{d x} d x & =\frac{1}{a(\tau)} \frac{d a(\tau)}{d \tau}[h(\tau) \\
& \left.-\int_{0}^{1} \sqrt{1-x^{2}} \frac{d f(x)}{d x} d x\right] .
\end{aligned}
$$

Substituting Eq. (14) in Eq. (12)

$$
\frac{d}{d \tau} a(\tau) \int_{0}^{1} \frac{x^{2} f^{\prime}(x)}{\sqrt{1-x^{2}}} d x=a(\tau) \frac{d h(\tau)}{d \tau} .
$$

The initial unloading slope is then given by, using Eq. (11)

$$
\begin{aligned}
\frac{d F}{d h}= & \frac{d F / d t}{d h / d t}=\frac{4}{1-\nu}\left[G(0) a\left(t_{m}\right)\right. \\
& \left.-\left.\frac{1}{v_{h}} \int_{0}^{t_{m}} \frac{d G}{d \eta}\right|_{\eta=t_{m}-\tau} \frac{d}{d \tau}\left[a(\tau) \int_{0}^{1} \frac{x^{2} f^{\prime}(x)}{\sqrt{1-x^{2}}} d x\right] d \tau\right] .
\end{aligned}
$$

When the unloading rate, $v_{h}$, is sufficiently fast, the second term on the right-hand side approaches zero. Once this limiting case is reached, Eq. (1) can be used to determine the "instantaneous" moduli, $G(0) /(1-\nu)$ or $E(0) /\left(1-\nu^{2}\right)$, provided that the contact depth, $h_{c}$ or area, $A$, is known as a function of $h_{m}=h\left(t_{m}\right)$. The latter condition is provided by Eq. (5) for axisymmetric indenters of arbitrary profiles, which becomes Eqs. (7) and (9) for conical and spherical indenters, respectively. Indeed, we have recently demonstrated the validity of Eqs. (5) and (16) for conical ${ }^{16,30}$ and spherical ${ }^{31}$ indentation in linear viscoelastic solids using finite element calculations.

In summary, we have derived a relationship between initial unloading slope, contact depth, and instantaneous relax- ation modulus for indentation in linear viscoelastic solids by a rigid indenter with an arbitrary axisymmetric smooth profile. This derivation shows that with increasing unloading rate, unloading slope converges to a limiting case given by Eq. (1). Thus, fast unloading is essential in determining the instantaneous modulus from the initial unloading slope using Eq. (1). Presently, very little attention is paid in the literature to the unloading rate when viscoelastic properties are measured using instrumented indentation techniques. This lack of attention to the unloading rate is believed to be the main cause for the reported disagreement with Eq. (1). Finally, it is evident from the derivation that Eq. (1) holds true for fast loading as well as for fast unloading since in both cases (i.e., $\left.\mp v_{h}\right)$ the second term in Eq. (16) approaches zero. The instantaneous modulus can thus be obtained by fast jumps during either loading or unloading for indentation in linear viscoelastic solids using axisymmetric indenters of arbitrary profiles.

The authors would like to thank Wangyang Ni, Mike Lukitsch, Yue Qi, Tom Perry, and Wes Capehart, Lou Hector, and Mark W. Verbrugge for valuable discussions. C.M.C. would like to acknowledge partial support from NSF of China, Project No. 10372101.

${ }^{1}$ E. H. Lee, Q. Appl. Math. 13, 183 (1955).

${ }^{2}$ J. R. M. Radok, Q. Appl. Math. 15, 198 (1957).

${ }^{3}$ E. H. Lee and J. R. M. Radok, J. Appl. Mech. 27, 438 (1960)

${ }^{4}$ S. C. Hunter, J. Mech. Phys. Solids 8, 219 (1960).

${ }^{5}$ G. A. C. Graham, Int. J. Eng. Sci. 3, 27 (1965).

${ }^{6}$ G. A. C. Graham, Int. J. Eng. Sci. 5, 495 (1967).

${ }^{7}$ W. H. Yang, J. Appl. Mech. 33, 395 (1966).

${ }^{8}$ T. C. T. Ting, J. Appl. Mech. 33, 845 (1966).

${ }^{9}$ T. C. T. Ting, J. Appl. Mech. 35, 248 (1968).

${ }^{10}$ L. Cheng, X. Xia, W. Yu, L. E. Scriven, and W. W. Gerberich, J. Polym. Sci., Part B: Polym. Phys. 38, 10 (2001).

${ }^{11}$ P.-L. Larrson and S. Carlsson, Polym. Test. 17, 49 (1998).

${ }^{12}$ S. Shimizu, T. Yanagimoto, and M. Sakai, J. Mater. Res. 14, 4075 (1999).

${ }^{13}$ M. Sakai and S. Shimizu, J. Non-Cryst. Solids 282, 236 (2001).

${ }^{14}$ M. Sakai, Philos. Mag. A 82, 1841 (2002).

${ }^{15}$ M. L. Oyen and R. F. Cook, J. Mater. Res. 18, 139 (2003).

${ }^{16}$ Y.-T. Cheng and C.-M. Cheng, Mater. Sci. Eng., R. 44, 91 (2004).

${ }^{17}$ M. F. Doerner and W. D. Nix, J. Mater. Res. 1, 601 (1986).

${ }^{18}$ G. M. Pharr, W. C. Oliver, and F. R. Brotzen, J. Mater. Res. 7, 613 (1992).

${ }^{19}$ W. C. Oliver and G. M. Pharr, J. Mater. Res. 7, 1564 (1992).

${ }^{20}$ B. J. Briscoe, L. Fiori, and E. Pelillo, J. Phys. D 31, 2395 (1998).

${ }^{21}$ M. R. VanLandingham, J. Res. Natl. Inst. Stand. Technol. 108, 249 (2003).

${ }^{22}$ S. P. Timoshenko and J. N. Goodier, Theory of Elasticity, 3rd ed. (McGraw-Hill, New York, 1970).

${ }^{23}$ A. E. H. Love, Q. J. Math. 10, 161 (1939).

${ }^{24}$ I. N. Sneddon, Int. J. Eng. Sci. 3, 47 (1965).

${ }^{25}$ C.-M. Cheng and Y.-T. Cheng, Appl. Phys. Lett. 71, 2623 (1997).

${ }^{26} \mathrm{H}$. Lu, B. Wang, J. Ma, G. Huang, and H. Viswanathan, Mech. TimeDepend. Mater. 7, 189 (2003).

${ }^{27}$ M. V. R. Kumar and R. Narasimhan, Curr. Sci. 87, 1088 (2004).

${ }^{28}$ W. N. Findley, J. S. Lai, and K. Onaran, Creep and Relaxation of Nonlinear Viscoelastic Materials (Dover, Mineola, 1989).

${ }^{29}$ G. T. Mase and G. E. Mase, Continuum Mechanics for Engineers, 2 nd ed. (CRC, Boca Raton, FL, 1999).

${ }^{30}$ Y.-T. Cheng and C.-M. Cheng, J. Mater. Res. 20, 1046 (2005).

${ }^{31}$ Y.-T. Cheng and C.-M. Cheng, GM Research and Development Center Publication R\&D-10, 059, February 21, 2005; Mat. Sci. Eng. A (in press). 